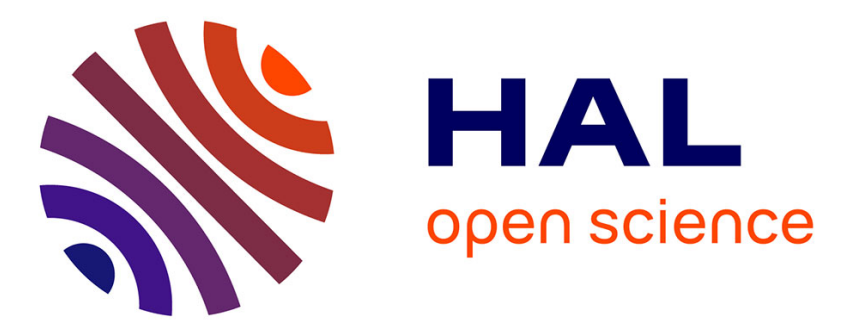

\title{
Experimental and theoretical study of a premixed vibrating flame
}

Françoise Baillot, Daniel Durox, Roger Prud'Homme

\section{To cite this version:}

Françoise Baillot, Daniel Durox, Roger Prud'Homme. Experimental and theoretical study of a premixed vibrating flame. Combustion and Flame, 1992, 88, pp. 149-168. hal-01995779

\section{HAL Id: hal-01995779 \\ https://hal.science/hal-01995779}

Submitted on 27 Nov 2020

HAL is a multi-disciplinary open access archive for the deposit and dissemination of scientific research documents, whether they are published or not. The documents may come from teaching and research institutions in France or abroad, or from public or private research centers.
L'archive ouverte pluridisciplinaire HAL, est destinée au dépôt et à la diffusion de documents scientifiques de niveau recherche, publiés ou non, émanant des établissements d'enseignement et de recherche français ou étrangers, des laboratoires publics ou privés. 


\title{
Experimental and Theoretical Study of a Premixed Vibrating Flame ${ }^{1}$
}

\author{
F. Baillot, D. Durox, and R. Prud'homme ${ }^{2}$
}

\begin{abstract}
An experimental study of vibrating flames above a cylindrical burner has been conducted in order to examine some of the fundamental characteristics of the flow perturbations-combustion interaction. Here the perturbation chosen is a space-time sinusoidal flow velocity. The vibrational nature of the flow has been studied with and without the flame, using a LDV setup and a tomography system. The prevalent kinematic phenomenon is an effect of flow pumping-indeed the velocity perturbation propagates along the vertical axis nearly at the mean flow velocity. A laminar flame in such a flow modulation $(<200 \mathrm{~Hz})$ responds with a periodic distortion around its steady, almost conical, configuration. The oscillation is the same frequency as that produced by the flow pumping. The deformation and the kinematics of the flame, studied by the means of a tomography system and a photographic record, are characterized by the height, the length, the area, and at the top by the flapping, the absolute speed, the thickness of the thermodiffusive zone, and the normal burning speed. In particular, the burning speed evolves globally between 0.5 and 2 times the laminar burning speed measured at the top of the reference steady flame. The area variations evolve from $11 \%$ to $25 \%$, for the RMS intensity of the velocity fluctuations well below $10 \%$. Nevertheless over a cycle the mean area is approximately equal to that of the laminar steady reference flame. On average the instantaneous stretching effects arc negligible on the front structure, in spite of the presence of large instantaneous effects like at the top. An analytical model, based on small disturbances theory and a constant burning speed, shows that the space-time front deformation results from waves originating at the flame base towards its upper limit. A comparison between theoretical and experimental results is in good agreement,
\end{abstract}

\section{NOMENCLATURE}

a amplitude of velocity perturbation

$c \quad$ propagation speed of velocity perturbation

$c_{1}, c_{2}$ speeds of progressive waves developed along the front

$f \quad$ frequency of the external sinusoidal perturbation

$f_{b} \quad$ frequency of the disturbance due to buoyancy effects of hot gases

$F \quad$ space-time function of the flame front position

$H$ height of the flame

$H_{0} \quad$ height of the steady flame in the reference state

j vertical vector

$k \quad$ velocity perturbation wavenumber $(=2 \pi f / c)$

$k^{*} \quad$ velocity perturbation wavenumber of reference $(=2 \pi f /\langle\mathrm{V}\rangle)$

$L \quad$ length of the tomographic flame front contour

$L_{0} \quad$ length of the tomographic steady flame front contour in the reference state

n unit normal vector at a point of the front directed towards the burned gases

$R \quad$ radius of the burner

$S \quad$ area of the flame surface

$S_{0} \quad$ area of the steady flame surface in the reference state

$S_{\mathrm{T}} \quad$ mean area over a cycle

$t \quad$ time

$T$ period of the external sinusoidal perturbation

$U$ horizontal velocity component in the (horizontal, vertical) polar coordinate system

$U$, derivative of the component $U$ with respect to $y$ at constant $t$

$v_{\mathrm{L}} \quad$ plane adiabatic normal burning velocity

\footnotetext{
${ }^{1}$ Publié dans Combustion and Flame 88: 149-168 (1992)

2 Laboratoire d'Aérothermique du CNRS, F92190 Meudon, France
} 
normal burning velocity at the top of the disturbed flame front

$v_{\text {no }} \quad$ normal burning velocity at the top of the steady flame front

$v_{\mathrm{T}} \quad$ mean burning velocity of the flame over a cycle

$v_{\perp} \quad$ component of the unburnt gases velocity normally to the front

v' RMS velocity

$V \quad$ vertical velocity component in the (horizontal, vertical) polar coordinate system

$V$, derivative of the component $V$ with respect to $y$ at constant $t$

$\bar{V} \quad$ mean vertical component

$\langle\bar{V}\rangle \quad$ average of the mean velocities $V$

$V_{\text {front }} \quad$ vertical velocity component of the unburned gases at the front crossing

$\bar{V}_{\text {front }}$ mean velocity of the instantaneous front velocities $V_{\text {front }}$

$V_{0} \quad$ vertical velocity of the theoretical uniform flow of reference

$x \quad$ horizontal coordinate of the polar system

$x_{1} \quad$ first term of the asymptotic development of $F$ with $a$

$y \quad$ vertical coordinate of the polar system

$y_{\mathrm{s}} \quad$ vertical coordinate of the front at the top

$Y \quad$ ordinate of a point in an inclined system attached to the front of reference

$w \quad$ normal flame speed of a point of the front $=\partial F / \partial t \cos \theta$

w absolute speed vector of a point of the front

$w_{s} \quad$ normal flame speed component at the top $\left[=(d y / d t)_{\text {top }}\right.$ with $\left.F\left(y_{s}, t\right)=0\right]$

\section{Greek Symbols}

a half-angle at the apex of the conic front of the steady theoretical flame of refeence

$\phi \quad$ velocity wave phase

$\lambda_{1}, \lambda_{2}$ characteristic wavelengths of the theoretical wrinkled flame

$\omega \quad$ phase speed of the velocity wave

$\theta \quad$ angle between the unit normal $\mathbf{n}$ and the horizontal at a point of the disturbed front of the theoretical flame

$\theta_{1} \quad$ first term of the asymptotic development of $\theta$ with $a$

$\Psi \quad$ progressive wave of kinematic disturbance

\section{INTRODUCTION}

The direct investigation of turbulent combustion waves remains a complex task, despite the advent of a new generation of computers and instrumentation, due to the random nature of the flows in which combustion takes place. Thus, in order to study some characteristics of the combustion-flow perturbations interaction, "simple" fundamental experiments involving the disturbance of a laminar flame have been conducted, in which the perturbation is considered to be well-known and well controlled:

(a) V-shaped flames disturbed by a street of vortices [1-4]

(b) vibrating flames,

(b1) stabilized upon a vibrating flame holder [4-6]

(b2) subjected to a time sinusoidal velocity field [3-7]

(b3) subjected to a time-space sinusoidal velocity field $[6,8,9]$

In the first case (a), the experimental contributions deal with the impact of vortices from a laminar or turbulent Von Karman street, created behind a cylindrical rod placed in an 
unburned gas flow, on a V-shaped flame front. This interaction can be identified with an idealized two-dimensional turbulence, localized in a precise part of the front, since the perturbation spectrum is reduced to the Strouhal frequency of the vortex passage. As in the case of turbulent flow fields, characterized by a large spectral distribution of different length scales, this phenomenon can be analyzed in terms of a relative scale defined as the ratio of the vortex length over the flame thickness. Since the first experiments [4], much work has concentrated on describing and analyzing the mechanisms along the front: the formation of undulations and cusps, the evolution of the wrinkling enlargement rate, the existence of a transverse propagative disturbance speed (which is equal to the velocity component of the unburned gas flow parallel to the front), and the differentiated role of the outer or inner vortices of the Von Karman street.

In the second type of experiments (b), a sinusoidal disturbance (low frequencies) is applied to the flame, either (bl) by directly vibrating the holder [6], or (b2) and (b3) by creating a flow modulation.

Experiments (bl) are similar to the case (a) where the perturbation is two-dimensional and localized on the flame front. The propagative laws of the front perturbations show some similar characteristics. The first observations made by Peterson and Emmons, when the disturbance amplitude is not too large, indicate the existence of a transverse wave that runs along the front at a speed $\mathrm{c}$ equal to the gas driving velocity parallel to the reference steady front. As for any $\mathrm{V}$-shaped configuration, the time evolution of the flame surface is difficult to obtain, because of the difficulty in defining the upper limit of the front, which is truly little luminous. Nevertheless a measurement of the area variations can be obtained for a given height limited by two steady planes.

In the case (b2), the flame is placed into a tube and subjected to a sinusoidal time modulation of the unburned gas flow, by using the frequency of the vertical duct analogous to a Helmholtz resonator [3, 7]. This problem becomes one of the interactive studies of the phenomena combining combustion and acoustics. The front dynamics has a similar behavior to that observed for the space-time modulation of the free flow. In Ref. 3 some steady contractions of finite thickness are observed along the front, similar to interference nodes with an amplitude that would be zero if there were no nonlinear effects. The presence of these nodes as well as the existence of a transverse wave with a speed $c$ have been demonstrated in a linearized calculation in Ref. 3 and a nonlinearized calculation in Refs. 8 and 10. As a reminder, we also mention some experimental and theoretical studies that have more specifically taken up the acoustic/combustion coupling by using the combustion chamber as a Helmholtz resonator [11-16]. These studies are initially based on some observations made by Rayleigh about the response of a diffusive flame to some standing waves developed in tubes [11]. Although they are similar to that of Boyer and Quinard [3] these studies concern purely acoustic parameters such as the power supplied by the flame and, with the exception of Ref. 12 , do not examine flame deformation with respect to the front shape and the surface.

The experiments of case (b3) are based on the existence of a space-time sinusoidal velocity field characterized by a wavenumber $k$ and frequency $f$. The selection of the sinusoidal modulation of the flow by a loudspeaker as a vibrating system makes it possible to isolate a single excitation frequency in a large band of use. Similarly, the disturbance amplitude is adjustable and reaches high values of velocity fluctuations, up to $30 \%$ of the mean flow velocity if desired. This problem corresponds more to an idealized combustion/ turbulence interaction because of the nature of the flow: the kinematic perturbations are convected at the speed $2 \pi f / k$, practically equal to the mean flow velocity which is similar to the case of a homogeneous turbulent flow with a constant mean velocity [17, 18]. The study bas been carried out on a conical flame. This configuration has the advantage of being a closed surface, and its top can be easily followed in time and space contrary to another point of the front (or a 
point of a V-shaped flame too) whose coordinates vary simultaneously in time. This geometry then gives access to parameters such as the instantaneous surface of the front and the normal burning velocity at the top.

The present article exhibits experimental results obtained by using new investigative and diagnostic techniques when a premixed flame is placed in such a space-time sinusoidal flow. These results are compared with those provided by the kinematic theory of the front deformation described hereafter.

\section{EXPERIMENTAL STUDY}

\section{Experimental Setup}

A premixed flame is stabilized above a burner with a convergent and cylindrical nozzle (Fig. 1) - for more details on the burner design, see Refs. 8, 19, and 25. Without any perturbation, the steady laminar flame is practically conical except at the top and in the anchorage zone (Fig. 2). Outside these two zones, the normal burning velocity $v_{L}$ is practically constant along the front $[19,22]$.

The study of the flame is carried out at low pressure $(0.25 \mathrm{~atm})$, which offers the following advantages:

1. A thickening of the thermodiffusive and reactive zones, allowing an easier visualization of the front thickness.

2. A better anchorage without flame holder.

3. The attenuation of an extraneous oscillation of the flame top, caused by a low-frequency disturbance $\mathrm{fb}(\approx 11 \mathrm{~Hz})$, induced by the action of gravity on the hot gases surrounding the flame [23-25]. Since the relative effects of buoyancy and pressure act in a similar way on the convection processes through the Grashoff number to that proposed in Ref. 26, the pressure reduction counteracts the disturbance action due to gravity.

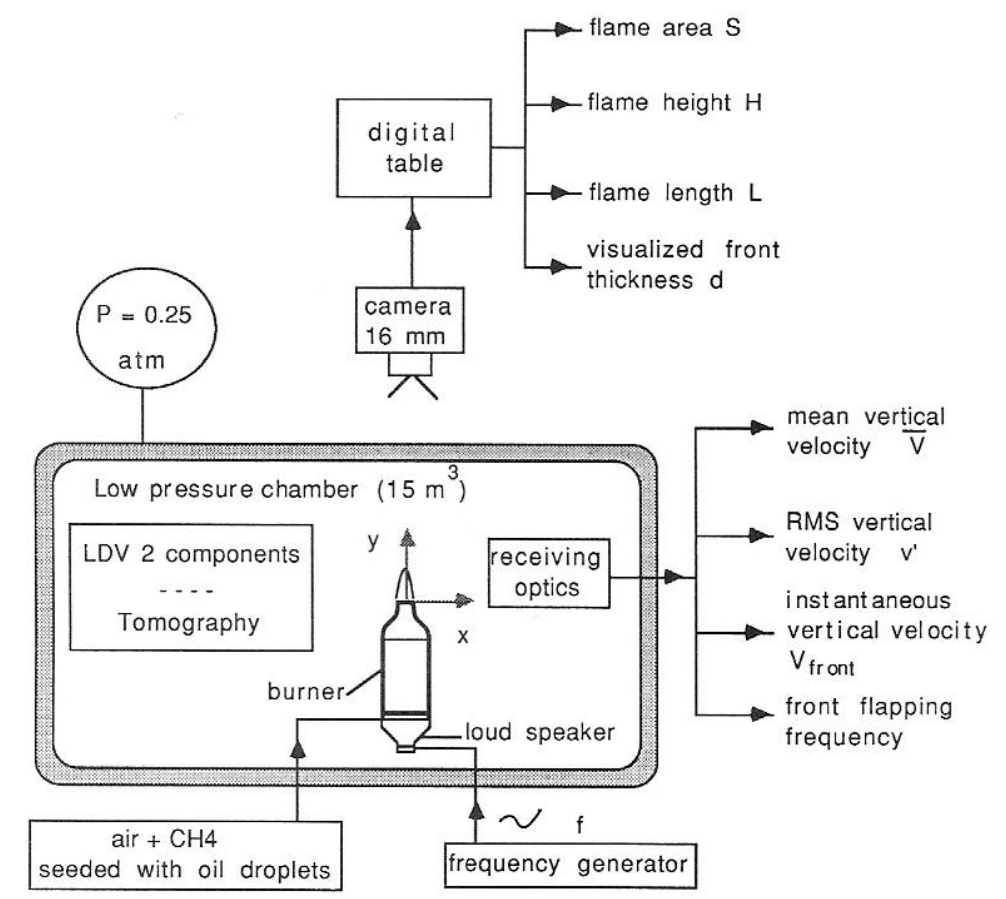

Fig. 1. Setup of the facility. 
An excitation of variable amplitude and low frequency, generated by the movement of the membrane of a loudspeaker placed at the bottom of the burner, causes the flow to vibrate. The membrane of the loudspeaker is made of fiberglass, resistant to any corrosive effects and ensuring impermeability to gases. The flame responds with a distortion and a periodical oscillation around its steady almost conical position. The intermittency zone thus swept by the flame appears to the eye as a thick front (Fig. 2).

The studied phenomenon appears only at frequencies less than a critical frequency $f_{c}$ whose the value depends on the pressure used $\left(f_{c} \approx 200 \mathrm{~Hz}\right)$.

For each frequency, two values of velocity fluctuation intensities of the perturbation have been chosen, which are, respectively, referred to as test 1 and test 2 . The experimental conditions are given in Tables 1 and 2.

The study deals as much with the kinematics of the vibrating flow as with the front deformation.

The investigation of the flow field has been accomplished utilizing a two-component LDV system. Since only those phenomena occurring in the unburned gases (including those just at the crossing of the flame front) were of interest, we have chosen to seed the flow with oil droplets (size less than $1 \mu \mathrm{m}$ ) that disappear just before the reactive zone. The selected seeding does not provide any Doppler signal in the gas zone with a temperature higher than the vaporization value of the oil droplets [27]. One of the channels (the green light beam) detects the front flapping. The intermittency in the reception of data causes a dropout in the P.M. signal, characterized by a plateau. The acquisition of the Doppler signal always occurred in the favorable situation where the rate of validated particles was at least 10 times higher than the excitation frequency $f$ of the generator, which avoids the degradation of the velocity signal-to-noise ratio [28]. The other channel (the blue light beam) was used to measure the axial component $V$ of the only unburned gas velocity from the analog signal of a counter. The signal was directly sent to an oscilloscope or a tape recorder. This technique is more precisely described in Ref. 29. Several pieces of information about the velocity were obtained:

Statistical data such as mean and RMS velocities.

Spectral data by a FFT analyser -HP 3582A characteristics: sampling frequency $=102.4$ $\mathrm{kHz}$, number of points used for each FFT $=256$, number of averaged data blocks $=64$ (if needed, possibility of averaging 256 data blocks).

Instantaneous velocities $V_{\text {front }}$ just before the crossing of the flame front inside the measurement volume. These velocity $V_{\text {front }}$ were measured by means of conditional sampling of the velocity signal: the instantaneous velocity was sampled just before the front crossing as the green light beam P.M. dropped out, since the analog output of the counter of the blue beam channel remained at the level of the last Doppler burst.

The investigation of the front deformation was performed using a laser tomography system. The droplets in the unburned gases vaporize at an isotherm of about $600 \mathrm{~K}$ in the flame thermodiffusion zone. When the flow is lighted by a laser sheet, only the droplets in the unburned gases scatter the light, the burned gases being transparent. The line of vaporization of oil droplet then indicates the position of the front [27]. In order to obtain the geometrical and kinematic characteristics of the front, we recorded its evolution using a 16-mm camera. From these sequences, filmed images have been processed by means of a digital table. The coordinates of front points are obtained with an accuracy between 0.05 and $0.1 \mathrm{~mm}$ and processed by a microcomputer. In addition to the visualization of this isotherm line, the reactive zone has been detected simultaneously, which has given, in particular, a value of the flame front thickness at the top. 


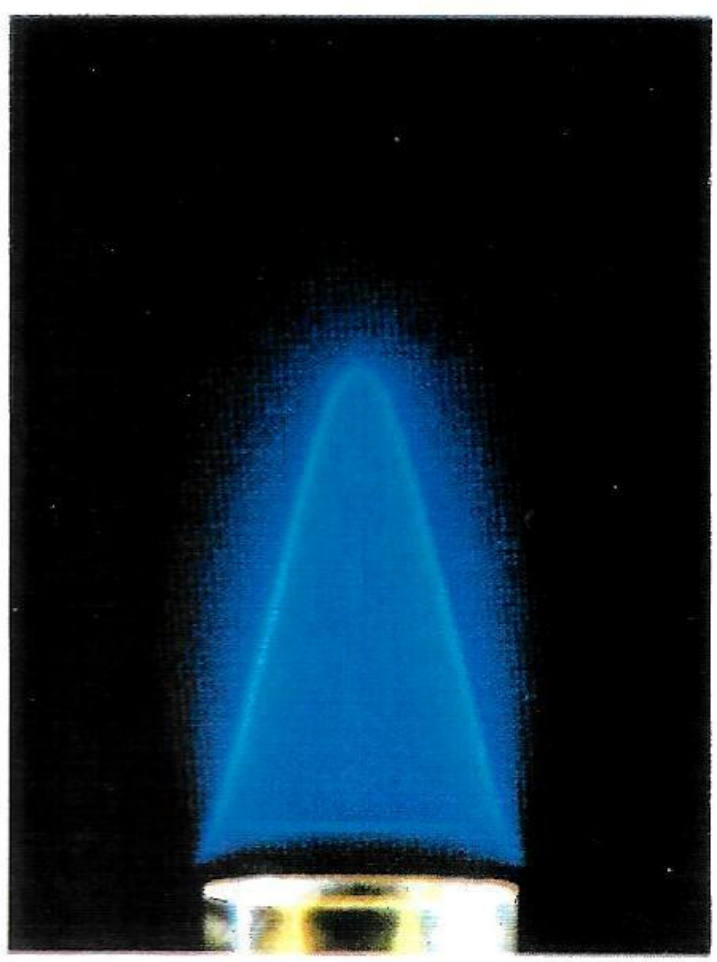

a

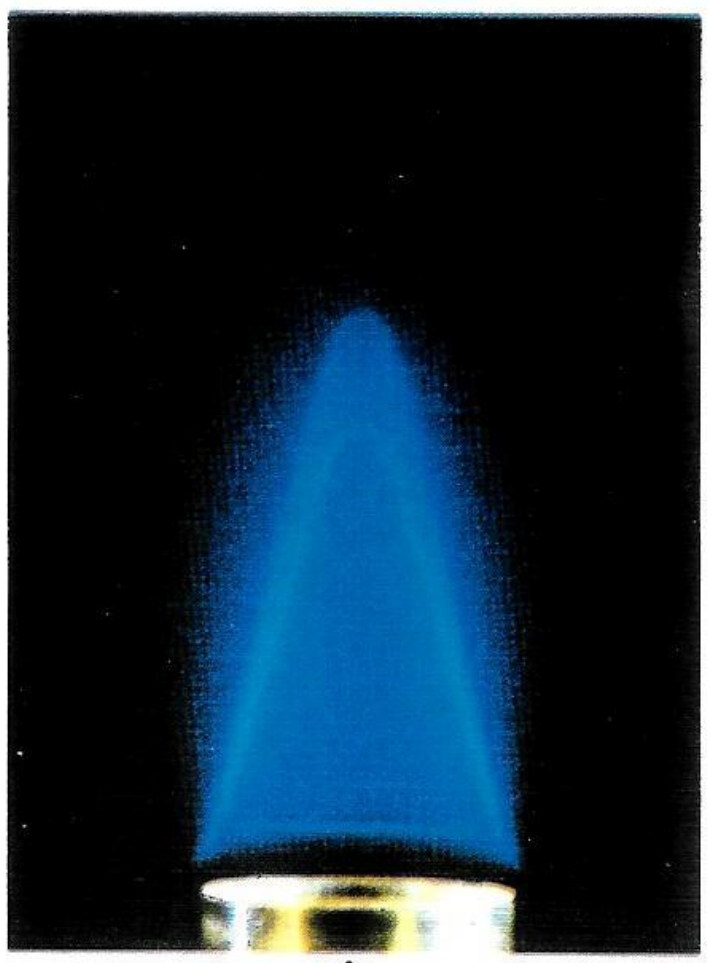

b

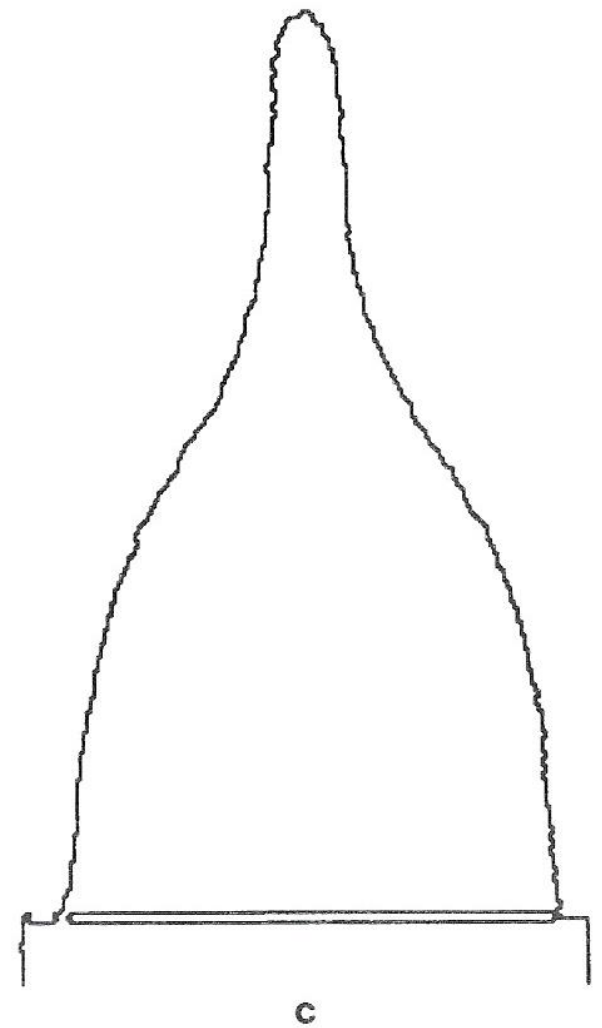

Fig. 2. (a) Laminar conical flame with no perturbation. (b) Thick front visible to the naked eye with $70.4 \mathrm{~Hz}$ perturbation. (c) Instantaneous wrinkled flame at $70.4 \mathrm{~Hz}$ (processed image). 
TABLE 1

\begin{tabular}{lc}
\hline Nature of the gases & Air + methane \\
Flow rate velocity of the mixture & \\
at the burner exit $(\mathrm{m} / \mathrm{s})$ & 1.94 \\
Equivalence ratio & 1.05 \\
Burner diameter $(\mathrm{mm})$ & 22 \\
Absolute pressure $(\mathrm{atm})$ & 0.25 \\
\hline
\end{tabular}

TABLE 2

\begin{tabular}{|c|c|c|}
\hline $\begin{array}{l}\text { Excitation frequency } f(\mathrm{~Hz}) \\
\text { of the generator }\end{array}$ & 35.2 & 70.4 \\
\hline RMS/mean exit velocity $v^{\prime} / \bar{V}(\%)$ & & \\
\hline $\begin{array}{l}\text { at } y=5 \mathrm{~mm} \text { Test } l \\
\text { RMS } / \text { mean exit velocity } v^{\prime} / \bar{V}(\%)\end{array}$ & 8.6 & 8.2 \\
\hline at $y=5 \mathrm{~mm}$ Test 2 & 6.1 & 5.3 \\
\hline
\end{tabular}

\section{Vibratory Nature of the Velocity Field with no Flame}

In the absence of flame, the undisturbed flow is steady, cylindrical, and spreads out slightly up wards. It showed no destabilization by vortices, caused by the shear layer between the jet and the ambient air, over a distance at least one and a half times longer than the space occupied by the flame. It is characterized by a nearly uniform distribution of the axial velocities over the whole jet cross-section except at the edge, in agreement with Ref. 19.

Disturbed by an external sinusoidal perturbation at the frequency $f$ provided by the loudspeaker, the jet, still with no flame, is characterized by some oblong structures starting at the base of the burner and moving at a speed $c$ (Fig. 3). The jet is defined by a sinusoidal velocity field, with the vertical component $V$ expressed by

$$
\begin{aligned}
& \text { at } x=0 \\
& \begin{aligned}
V(y, t)= & \bar{V}(y) \\
& +\sqrt{ } 2 v^{\prime}(y) \sin (2 \pi f t-\phi(y)),
\end{aligned} \\
& \text { with } \phi(y)=k y+\phi_{0},
\end{aligned}
$$

where $x$ and $y$ are, respectively, the horizontal and the vertical polar coordinates (Fig. 1), $v^{\prime}$ is the RMS intensity of fluctuations, $\bar{V}$ is the mean velocity, $\phi$ is the wave phase angle between the velocity fluctuations and the initial vibration of the generator, $f$ is the forcing frequency, and $k$ is the perturbation wavenumber related to the propagation velocity $c$ by e $=2 \pi f / k$. We have also verified, as De Soete did, that the $V(y, t)$ component at the burner exit was indeed sinusoidal and that the phase angle remained constant in the planes $y=2$ or $5 \mathrm{~mm}$, as far as the measurement domain remained outside the boundary layer of the jet.

In Fig. 4 , the evolution of $\left(\phi-\phi_{0}\right) / k^{*}$ versus $y$ has been plotted. The parameter $\mathrm{k}^{*}$ is the wavenumber linked to the propagation speed $(\bar{V})$ of the velocity perturbation, where $(\bar{V})$ is the average of the mean velocities $V(y)$, as defined by the equality 
$\left(\bar{V}=\sum_{i=1, N} \bar{V}\left(y_{l}\right) / N\right) \quad(\mathrm{N}$ is the number of points used to estimate $(\bar{V}))$. Whatever the flow conditions (frequency and average velocity), the results are well grouped around a unique straight line of interpolation calculated by a least squares method.

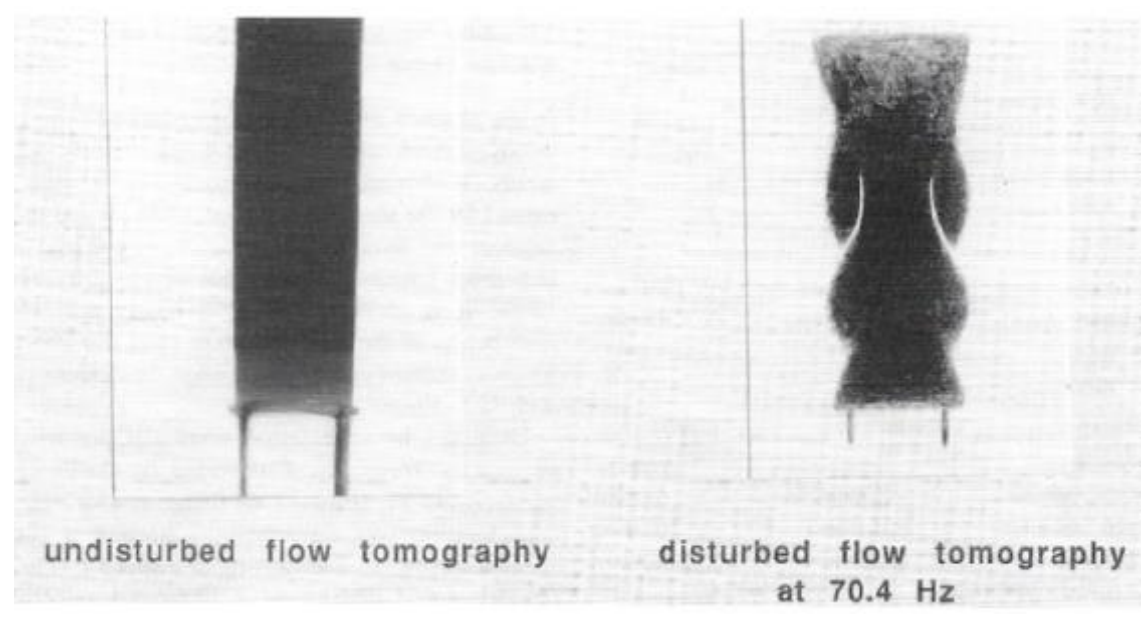

Fig. 3. Shape of a meridian section of the undisturbed and disturbed flows with no flame by laser tomography.

With no flame at $70.4 \mathrm{~Hz}$ as at $35.2 \mathrm{~Hz}$, the speed $c$ of the velocity wave along the axis remains lower by about $33 \%$ than the average $\langle\bar{V}\rangle$. A similar study carried out with a hot wire anemometer confirms the present results [6]. These data are also shown in Fig. 4.

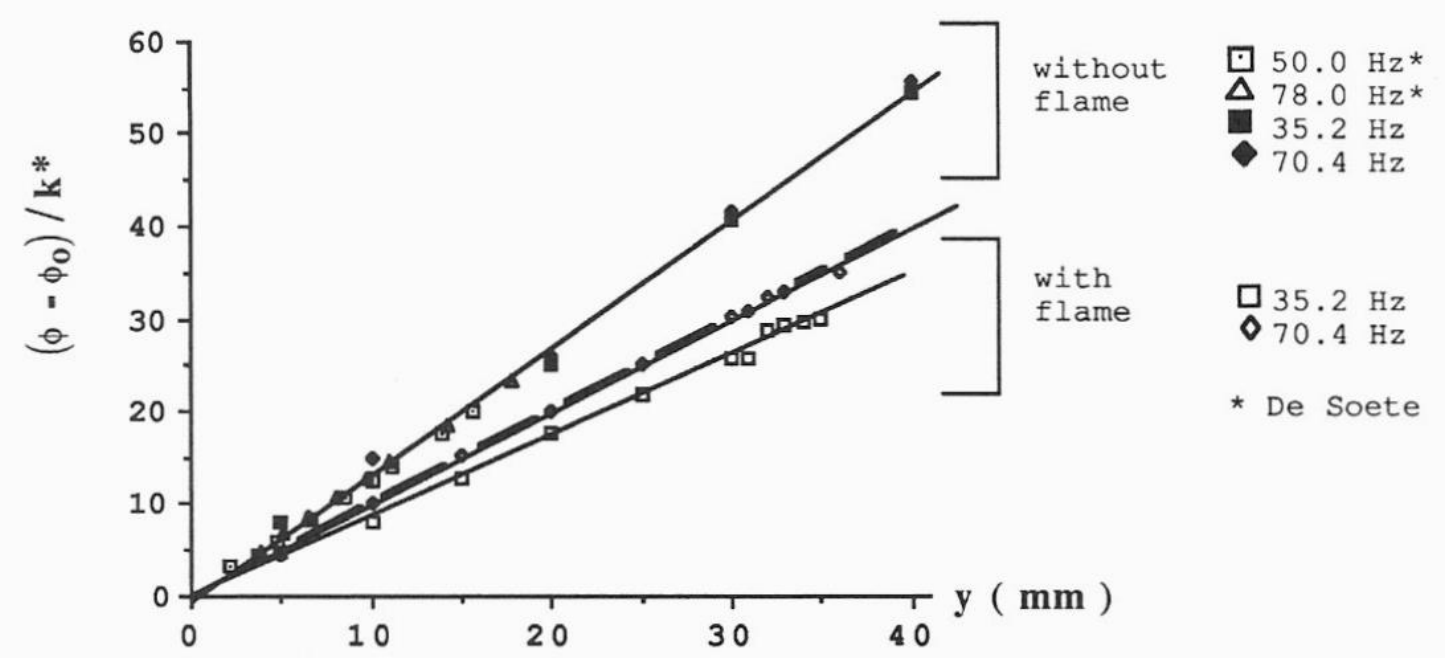

Fig. 4. Evolution of the phase angle $\phi$ of the velocity perturbation versus $y$ in the absence and in the presence of flame. - , straight line of interpolation; - - straight line characterizing the propagation at the average speed $\bar{V}$.

This space-time velocity disturbance assumes that the prevalent phenomenon of the flow field is not as much an acoustic disturbance as an effect of forcing fluid, causing a space-time pumping of the flow. Of course, the disturbance created near the loudspeaker which 
propagates inside the cylindrical burner is of acoustic type as shown by the measurements of the mean axial velocity carried out by De Soete [6], ( $\phi=$ const inside the burner whatever the $y$ value). Outside the burner, however, there is an adaptation of the flow to new boundary conditions evidenced by the existence of this phase angle $\phi(y)$.

\section{Velocity Field with Flame}

The velocity measurement technique with oil droplets does not allow measurement of the velocity of the burned gases, and therefore two zones of study may be investigated:

1. The zone of only unburned gases situated inside the conical envelope of the front flapping.

2. The intermittent zone.

\section{Unburned Gases Zone}

The spectral study of the velocity signal (Fig. 5) and its correlation with the generator signal show that the velocity component $V$ can be correctly represented in the exit plan of the burner $(y=2 \mathrm{~mm})$ by Eq. 1 , as long as the domain of measurement is outside the boundary layer, equivalent to $10 \%$ of the exit cross-section where in fact $v^{\prime}, V$, and $\phi$ depend on the horizontal abscissa $x$.

The velocity measurements along the vertical axis of symmetry show that $V(y, t)$ still follows closely this sinusoidal evolution represented by Eq. 1, except in the vicinity of the intermittent zone. In fact, in this region the low frequency $f_{b}$ of the sinusoidal disturbance due to buoyancy (see $\S$ Experimental Setup) can be detected: in Fig. 5, from $y=25 \mathrm{~mm}$ at $35.2 \mathrm{~Hz}$ and from $y=20 \mathrm{~mm}$ at $70.4 \mathrm{~Hz}$, the spectral analysis of the velocity signal shows the two predominant frequencies equal to $f$ and $f_{b}\left(f_{b}=10.9 \mathrm{~Hz}\right.$ at $35.2 \mathrm{~Hz}$ and $f_{b}=11.2 \mathrm{~Hz}$ at 70.4 $\mathrm{Hz}$ ). However, in the nonintermittent zone, since the amplitude of the disturbance remains very small with respect to the intensity of the forced kinematic fluctuations of the movement, the Eq. 1 remains a good approximation of $V(y, t)$ (Fig. 6).

The cross-correlation between the generator signal and the local velocity signal makes it possible to obtain $\phi$; the evolution $\phi(y)$ is plotted in Fig. 4 (the method used to obtain $\phi$, is the same as the one with no flame). The phase angle $\phi$, still varies linearly with $y$, but the value of the slope of the corresponding straight line of interpolation, that is, the nondimensional wavenumber $k / k^{*}$ us not the same as before: $k / k^{*}=0.88$ at $35.2 \mathrm{~Hz}$ and $k / k^{*}=0.98$ at $70.4 \mathrm{~Hz}$ instead of 1.33 .

Therefore the presence of the flame modifies the propagation speed but does not change its nature since the velocity fluctuations remain a sinusoidal function of the frequency $f$ and the wavenumber $k$. This modification can be explained both by hydrodynamic interaction of the wrinkled flame with the incident flow and by expansion of the unburned gases within the thermodiffusive zone. This influence leads to a propagation speed $c$ that is practically equal to the average $\langle\bar{V}\rangle$ of the mean flow velocities.

In Fig. 6, the mean velocity $\bar{V}(y)$ and the intensity $v$ ' of the fluctuations decrease by $3 \%$ and by about $30 \%-40 \%$, respectively, in presence of the flame, as shown by the measurements carried out along the axis. All the discrepancies observed in the cases with and without the flame can be interpreted as a display of the hydrodynamic effects of the flame upon the flow which retroacts" by diminishing the velocity amplitude.

At the burner exit $(0<y<10 \mathrm{~mm})$, the slope of the velocity $\bar{V}$ is not at all flat, as in the case with no flame; the velocity is greater at the exit and decreases with increasing $y$. This phenomenon may be due to a nozzle effect caused by the heating of the burner which is 
attenuated with $y$. This has been already observed in a laminar steady case [30] and in a turbulent case as well [31]. For $y \geq 10 \mathrm{~mm}$, regard of excitation frequency used, an increase is observed, possibly due to expansion effects of gases.

In Fig. 6 we can notice a clear decrease of the fluctuation $v$ ' in the field of unburned gases as the distance from the burner increases. Then, just before the flapping zone, there is a large and sudden increase of $v^{\prime}$, with a maximum at the entrance of the intermittency zone. This $v$ 'increase zone extends over 3-10 times the thermodiffusive thickness of the flame. This effect increases with the frequency $f$ which bas to be compared with the front kinematics whose movement becomes wider from 35.2 to $70.4 \mathrm{~Hz}$. This phenomenon seems to be a turbulence increase just upstream of the flame and is probably due to a hydrodynamic influence of the flame upon the flow.

Intermittent Zone. It should be remembered that the oil droplets, used to measure the velocities, disappear at an isotherm before the reactive zone and give information only in the unburned gases. Therefore the analog velocity signals coming out of the counter present some plateaus, due to the absence of luminous signal. The duration of these plateaus increases as far as the measurement volume approaches the upper limit of the intermittent zone. The counter is then held until the arrival of the next oil droplet in the measurement volume. Such recordings of analogic velocity signals are presented in Ref. 29.
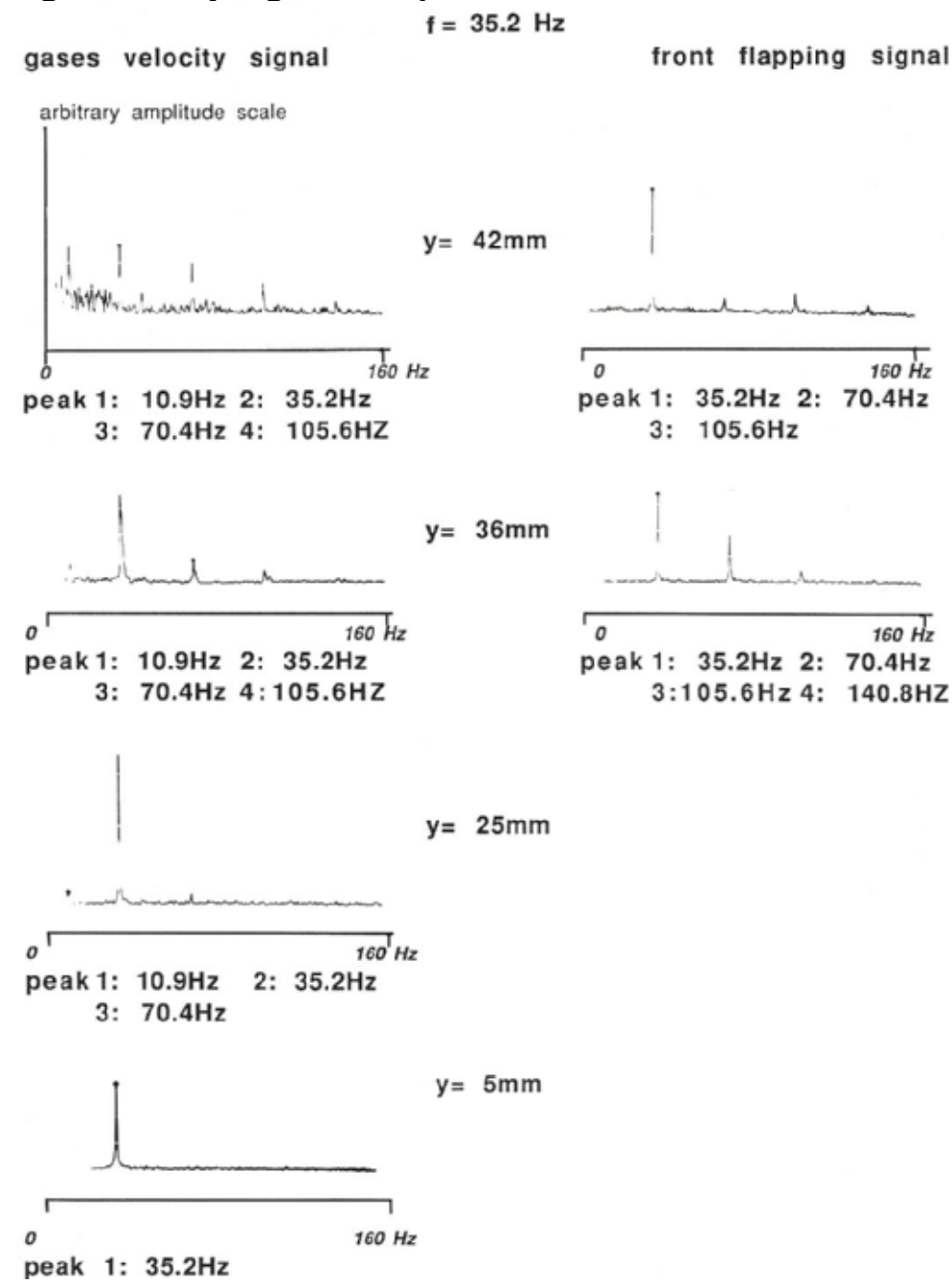

Fig. 5. Frequency spectra of the analog velocity signals and of the front flapping signals along the $y$ axis at $x=0 f=35.2 \mathrm{~Hz}$ (test 1$)$. 

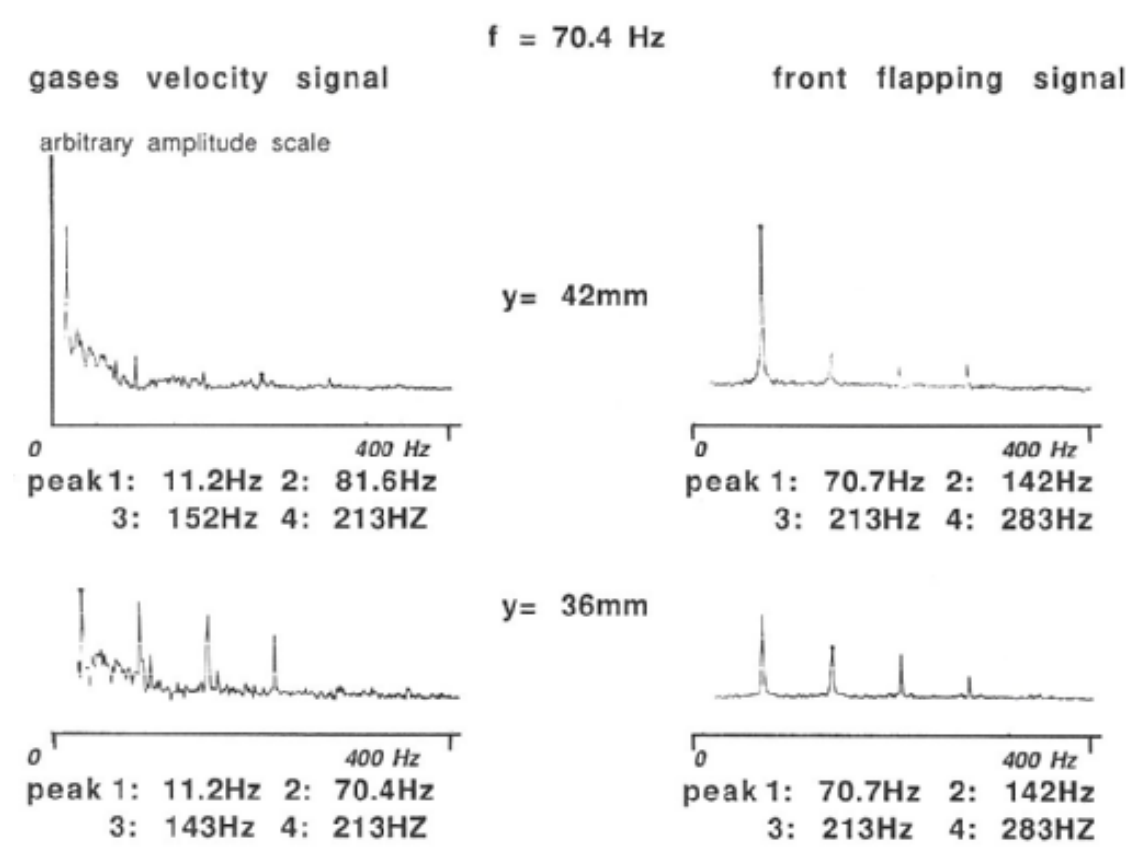

$y=36 \mathrm{~mm}$
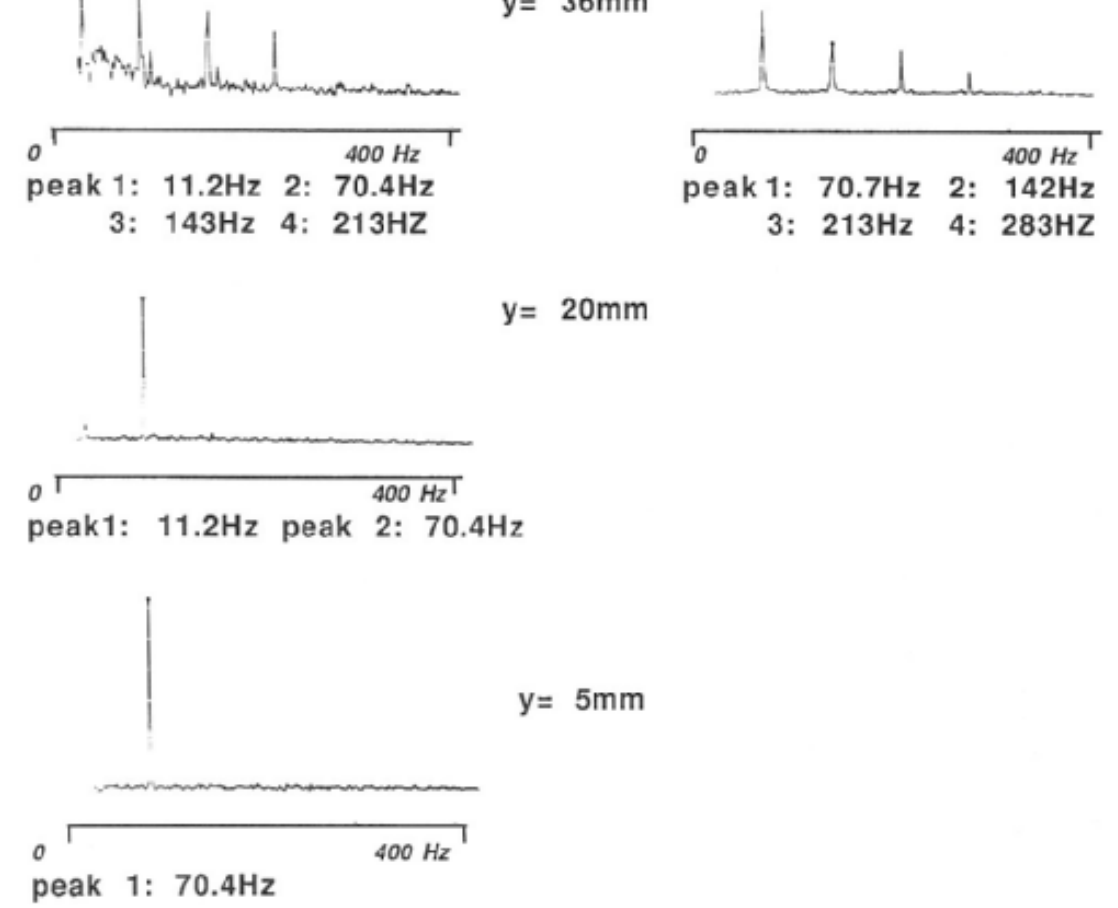

Fig. 5. $f=70.6 \mathrm{~Hz}$ (Continued)

The spectral analysis of the velocity signal always shows the two predominant frequencies equal to $f$ and $f_{b}$ (see previous section). However, at $35.2 \mathrm{~Hz}$ the signal maintains the same regular shape as in the first zone; the amplitude of the "disturbance $f_{b}$ " remains lower than that of the "excitation $f$." The front and the velocity signal are in phase. On the other hand, at

$70.4 \mathrm{~Hz}$, the velocity signal [8] is somewhat modified, in particular, by a superposition of a nonnegligible modulation of the low-frequency $f_{b}$ perturbation (see in Fig. 5 the amplitudes corresponding to $f$ and $f_{b}$ ). This phenomenon leads to a fluctuation of the value of the unburned gas velocity $V_{\text {front }}$ at the crossing of the front which increases with $y$. Therefore the mean value $\bar{V}_{\text {front }}$ calculated over time at a point of the front (Fig. 7), tends towards the mean velocity $\bar{V}$ measured at this same position (Fig. 6), when the frequency is increased from 35.2 to $70.4 \mathrm{~Hz}$. Moreover the frequency of the vibrating flow shows a discrepancy compared to the value $f$, which increases with $y$. The front flapping and die velocity signal do not evolve completely in phase. A display of this distortion is given by the measurement of $\bar{V}_{\text {front }}$ in Fig. 7: the maximum $\left(\bar{V}_{\text {front }}\right)_{\max }$ is no longer obtained when the flame is the tallest, as $f$ increases 
(this was confirmed at another frequency $(106 \mathrm{~Hz})$ ); on the contrary, the associate position $y_{\max }$ moves away from the highest position $H_{\max }$ of the front.

In order to carry out a more precise quantitative study of the velocity signal starting from the mean and fluctuating velocities measurement, a conditional sampling would be necessary, taking into account only the velocities of the unburned gases, that is, excluding the plateaus. Therefore in die special case of $v^{\prime}$ (Fig. 6), without such an operation, we observe a large decrease, followed at the end of the intermittency zone by a rise.
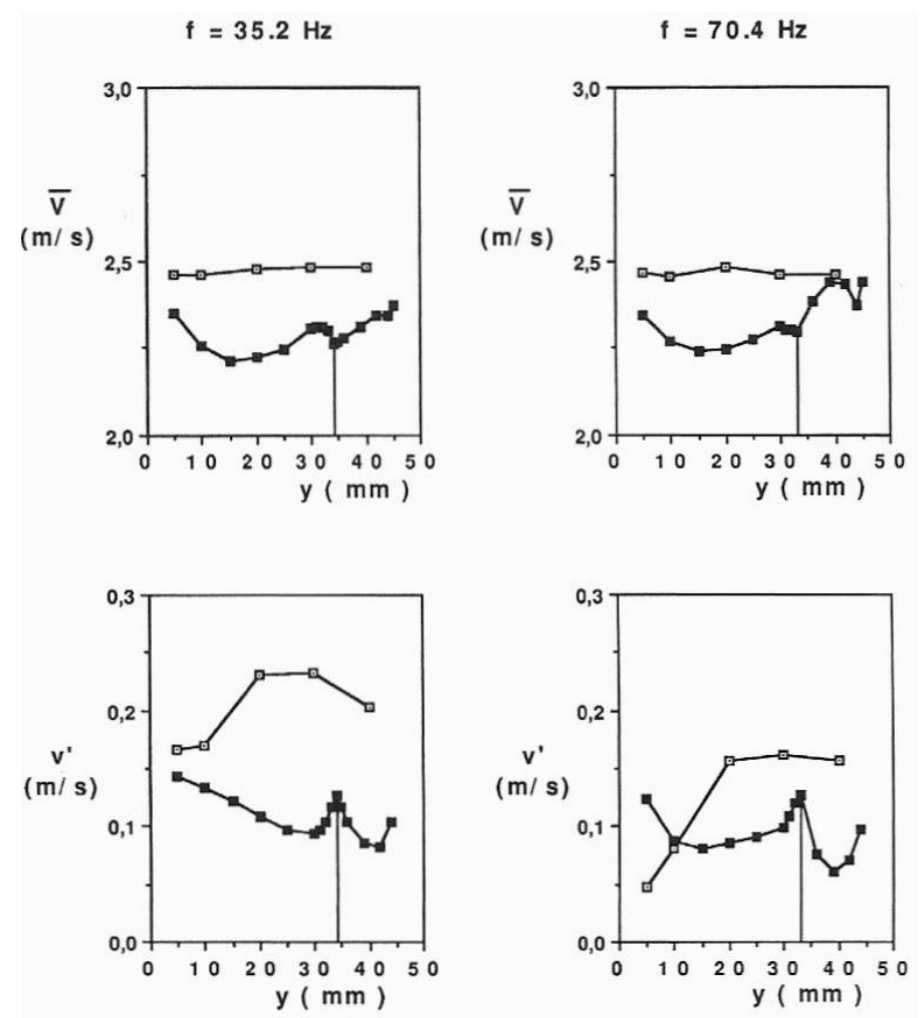

Fig. 6. Evolution of the mean velocity and rms fluctuations versus $y$ in the absence $(E)$ and in the presence $(\bullet$ ) of flame at $\mathrm{x}=0$. , limit of the intermittency zone (test 1$)$.

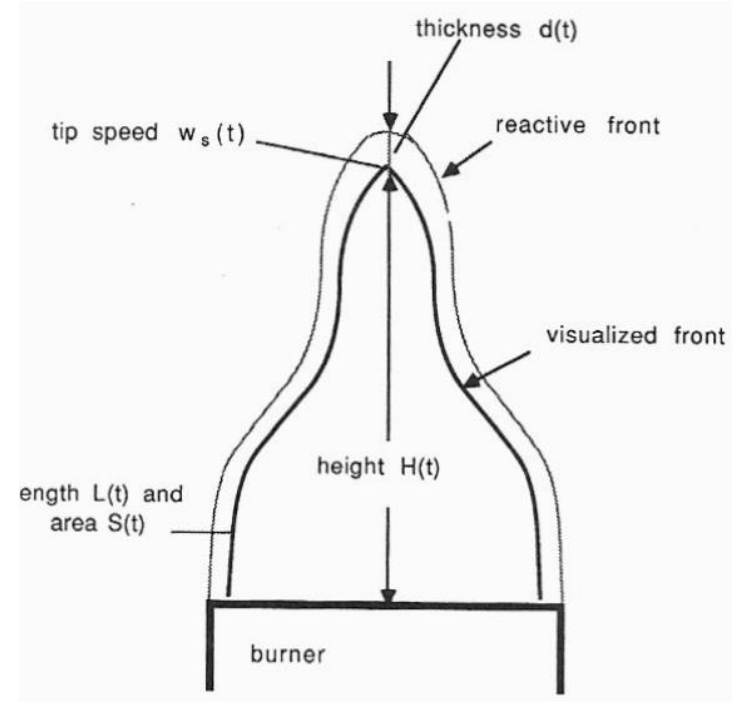

Fig. 8. Definition of the different flame parameters. 
This sudden increase may be explained by the hydrodynamic effect due to the unsteady movement of the front, and more particularly at the top zone when the flame is the tallest. In particular, at $70.4 \mathrm{~Hz}$, the flame is "pinched" close to the top, so that the combustion is practically instantaneous and cuts off the flame (a complete front deformation cycle is shown in Ref. 8).

\section{Deformation and Kinematics oh the Flame Front}

As soon as the laminar flame is subjected to the disturbed flow, it moves with an elongation phase and a collapse phase on both sides of its reference steady state. The spacetime evolution of this deformation, as compared with the reference steady state $(.)_{0}$ is characterized by the height $H(t)$, the length $L(t)$ of the front contour corresponding to the projection of the front surface in a vertical plane of symmetry, the axisymmetrical area $S(t)$, and at the top by the flapping nature, the absolute flame speed $w_{\mathrm{s}}(t)$ and the thickness of the visualized thermodiffusive zone (Fig. 8). The parameters of the steady state of reference are reported in Table 3.

The spectral analysis of the analog signal of the top flapping shows an adaptation of the flame to the upstream disturbance: the front flapping is periodical, with a frequency identical to that of the loudspeaker vibration at $35.2 \mathrm{~Hz}$. Nevertheless we observe a slight difference (less than $0.5 \%$ ) between the front flapping and the loudspeaker vibration, when the frequency of the loudspeaker is equal to $70.4 \mathrm{~Hz}$. This is probably due to the disturbance induced by the buoyancy effects in the gases $[8,25]$. Moreover, the frequency $f_{b}$ of the buoyancy effects disturbance does not clearly appear on the flapping spectrum, as is the case for the velocity signal. Therefore the sensitivity of the front to the latter remains small, contrary to the velocity field (see the previous section). Thus the flame is more sensitive to the main disturbance started at its base than to the presence of the disturbance of the frequency $f_{b}$. The front is then wrinkled essentially by a progressive wave that propagates from its base up to its top.

The measurement of the distances between two successive undulations is not identical in time. Thus it is impossible a priori to define a single propagation speed. However, the existence of a propagation velocity close to the velocity of the mean flow represents the phenomenon, as suggested in Refs. 4 and 6. This result is also advanced in Refs. 1-3. The model proposed at the end of this article confirms this for a flame that is sufficiently wrinkled or tall [8]

TABLE 3

Flame Characteristics in the Reference Steady State for Each Experimental Case

\begin{tabular}{cccc}
\hline$f(\mathrm{~Hz})$ & $H_{0}(\mathrm{~mm})$ & $L_{0}(\mathrm{~mm})$ & $S_{0}\left(\mathrm{~mm}^{2}\right)$ \\
\hline 35.2 & 39.7 & 83.3 & 1527 \\
70.4 & 39.6 & 83.1 & 1505 \\
\hline
\end{tabular}

In Fig. 9, an increase of the flapping amplitude $\left(H_{\max }-H_{\min }\right) / \mathrm{Ho}$ is observed with the amplification of $\left(v^{\prime} / \bar{V}\right)_{y=5 \mathrm{~mm}}$ or when the frequency $f$ is increased from 35.2 to $70.4 \mathrm{~Hz}$. Nevertheless its evolution is linear neither with $a$ nor with $f$. Overall, as shown in Fig. 10, the 
flame has a mean elongation of $\pm 15 \%$ on both sides of its reference position. This is important in comparison with the ratio $\left(v^{\prime} / \bar{V}\right)_{y=5 \mathrm{~mm}}$ which remains much lower than $10 \%$ (Table 2). However, during a cycle, the duration of the elongation phase is systematically longer than that of the collapse phase. At $70.4 \mathrm{~Hz}$, a sudden transition from $H_{\max }$ to appears due to a strong elongation effect at the top. It seems that this phenomenon is an inherent characteristic of the excitation frequency $f$ and is independent of the disturbance amplitude within the experimental approximation.

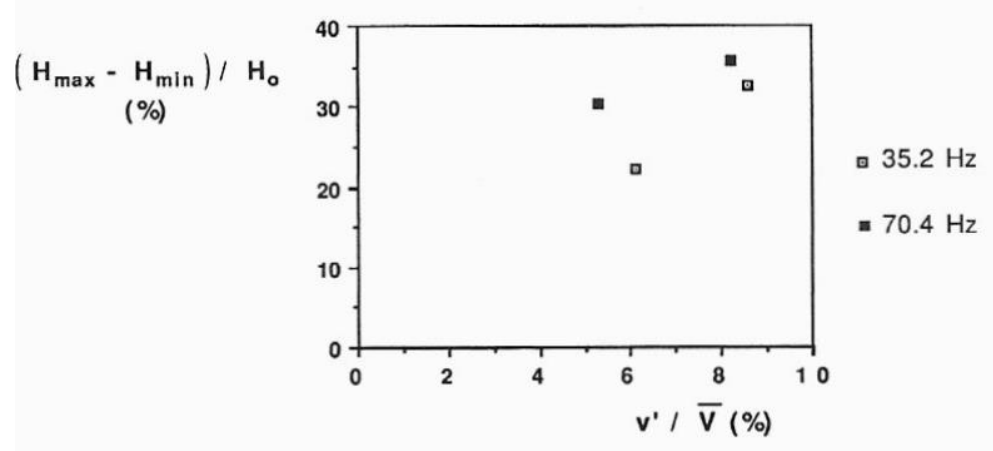

Fig. 9. Evolution of the tip flapping $\left(H_{\max }-H_{\min }\right) / H_{0}$ versus $\left(v^{\prime} / \bar{V}\right)_{y=5 \mathrm{~mm}}$ and $f$.

We have measured the length $L(t)$ of the contour and deduced the area $S$ of the flame.

In Fig. 10, it appears that the variations of $H$ and $L$ are similar, regardless of frequency. This strong correlation between $H$ and $L$ is due to the absence of large frontal distortions.

The comparison of the curves $S(t)$ and $H(t)$ shows that the flame essentially evolves in the elongation phase (Fig. 10): the area increases during about $60 \%$ of the cycle at $35.2 \mathrm{~Hz}$ and $70 \%$ at $70.4 \mathrm{~Hz}$; the height increases during about $55 \%$ of the cycle at $35.2 \mathrm{~Hz}$ and $75 \%$ at $70.4 \mathrm{~Hz}$. We observe that the height extrema are a lagging behind those of the area. This seems to be due essentially to the kinematic effects, as suggested in Ref 8 .

The area variation reaches $25 \%$ at $35.2 \mathrm{~Hz}$ and $11 \%$ al $70.4 \mathrm{~Hz}$, for an intensity ( $v$ ') $\bar{V})_{y=5 \mathrm{~mm}}$ of $6.1 \%$ and $5.3 \%$, respectively (Fig. 10). During a cycle, the mean area $S_{T}$ of these instantaneous area measurements is approximately equal to the area of the reference laminar flame $S_{0}$ (the difference is less than 5\%). It follows that the front propagates on average at a relative burning velocity $v_{T}$ equal to the adiabatic laminar burning velocity. In a purely turbulent flow condition, the same conclusion was reached in Ref. 32. The instantaneous stretching effects due to the front curvature and to the deformation rates would therefore be negligible on average on the front structure, or at the most, smaller than the measurement uncertainties. Of course these effects may be locally strong, as shown by the results of the evolution of the top normal burning velocity presented below.

From the absolute speed $w_{s}$ of the flame top, taken from the $H(t)$ curve (Fig. 10), the normal burning velocity $v_{n}$, at the top bas been calculated, with respect to the instantaneous velocity at the front crossing. The variables at the front top are defined as follows:

$$
\begin{array}{ll}
* & v_{n}=V_{\text {front }}-w_{s}, \\
* & w_{s}=d H / d t .
\end{array}
$$

The velocity $v_{n}$ is presented in Fig. 11: it evolves globally between 0.5 and 2 limes the laminar burning velocity $v_{\text {no }}$ measured at the top of the reference associated steady flame (thus $v_{\text {no }}$ takes into account the curvature effects, and is therefore different from the plane adiabatic burning velocity $v_{L}$ ). The values of $v_{n}$, may exceed $2 v_{\text {no }}$ during elongation but also can decrease strongly and tend toward zero. The average $\bar{v}_{n}$ of $v_{n}$, over a cycle becomes 
clearly smaller than $v_{\text {no }}$ at $70.4 \mathrm{~Hz}$, if it remains equal to $v_{\text {no }}$ at $35.2 \mathrm{~Hz}$. The top stretching effects therefore increase, on average, with frequency and tend to reduce the burning velocity.

The superposition of curves $S(t)$ and $v_{n}(t)$ (Figs. 10 and 11), shows the coincidence of the minima (maxima) of $v_{n}(t)$ with the maximum (minimum) areas of the instantaneous surfaces. Thus, when the flame is strongly elongated (collapsed), the stretching effects, tangential to the front, can impose a considerable decrease (increase). Similar observations have been formulated in Ref. 6.
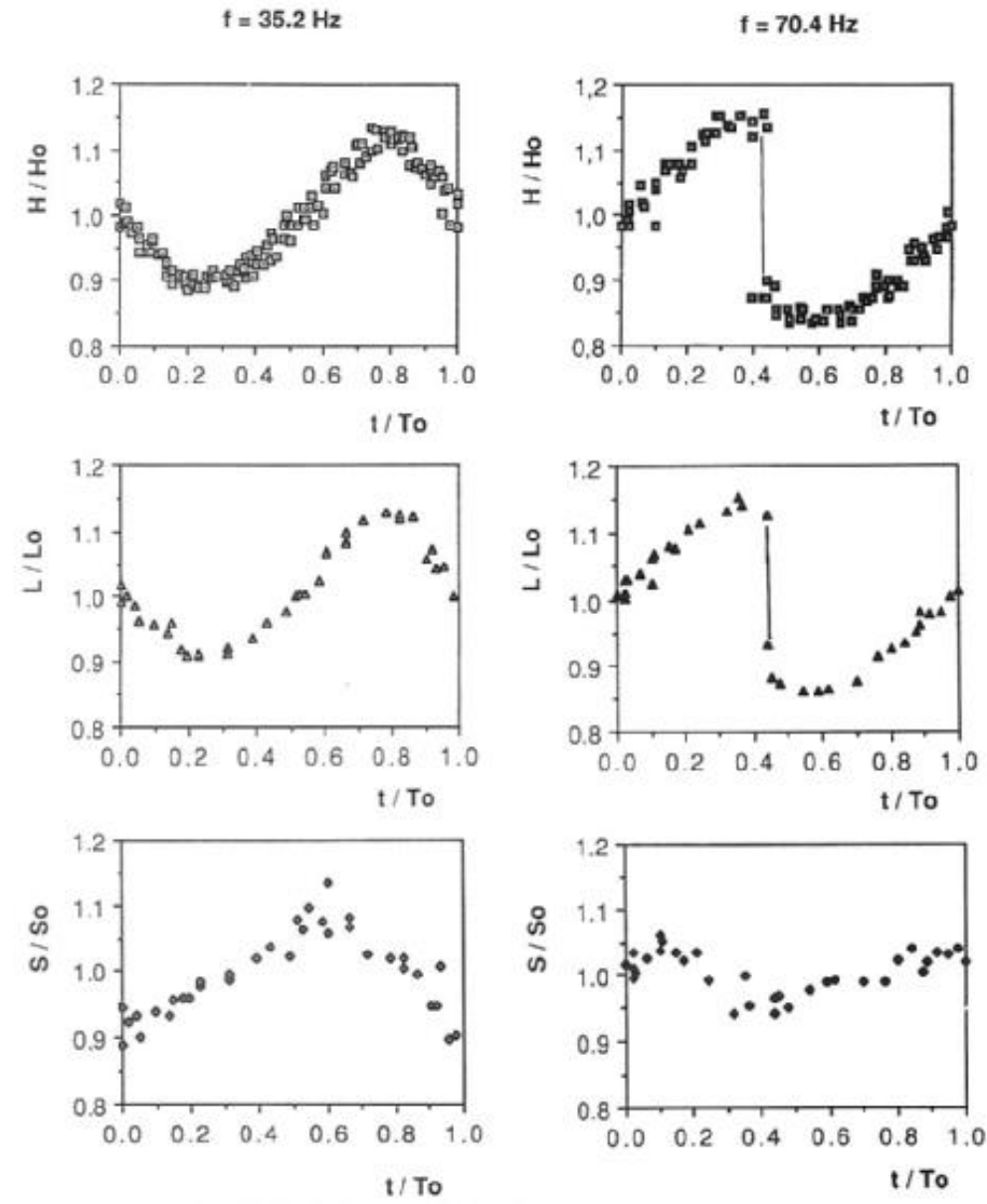

Fig. 10. Evolution of height, length, and area versus time (test 2).
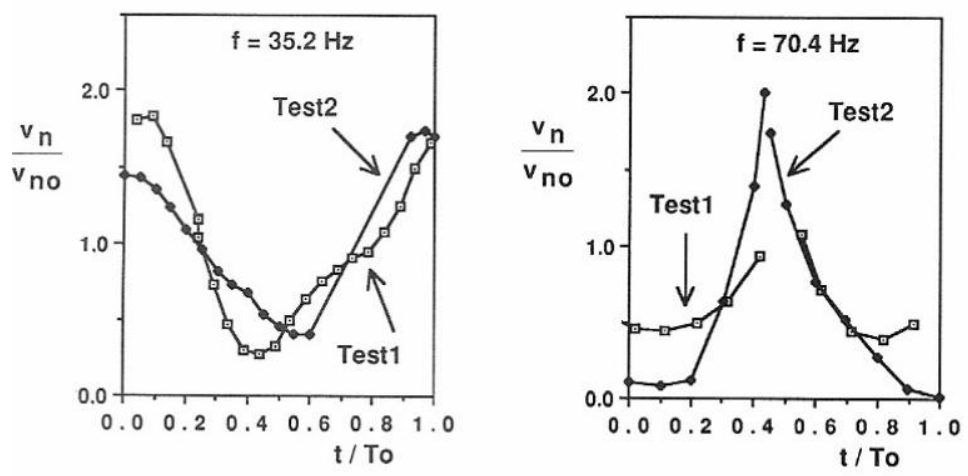

Fig. 11. Evolution of the normal burning velocity at the top versus time $v_{n o}: 2.31 \mathrm{~m} / \mathrm{s}$ (test 1$) ; 2.32 \mathrm{~m} / \mathrm{s}$ (test 2 ). 
Lastly, during test 1 it was possible to measure the time evolution of the top, visualizing the external part of the reactive zone (Fig. 12). This point moves in an analogous manner to that of the last visualized isotherm of the thermodiffusive zone used as reference for the height calculation. Since the distance $d$ that separates them remains of the same order of magnitude over a cycle, it can be said that the flame thickness at the top is almost uniform. We have $d \approx 1 \mathrm{~mm}$, a value similar to that of the characteristic distance of the steady flame. Thus low frequencies have no in fluence upon the combustion processes for a first-order approximation.

This experiment clearly shows the flame/flow coupling.

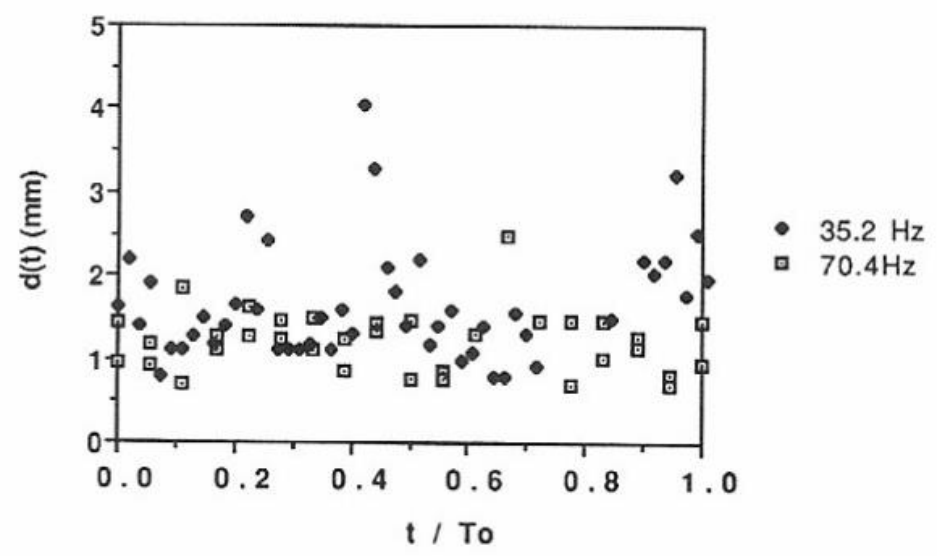

Fig. 12. Evolution of the visualized thermodiffusion thickness versus dine.

\section{THEORETICAL STUDY}

In order to investigate this behavior, we have analytically studied the response of a thin premixed flame stabilized above a burner with a circular cross-section. The computation has been extended to the case of a rectangular geometry, that is, to a dihedric or a V-shaped flame [8].

The configuration of the reference steady flame placed in a uniform flow of velocity $V_{0} \mathbf{j}$ is conical (Fig. 13).

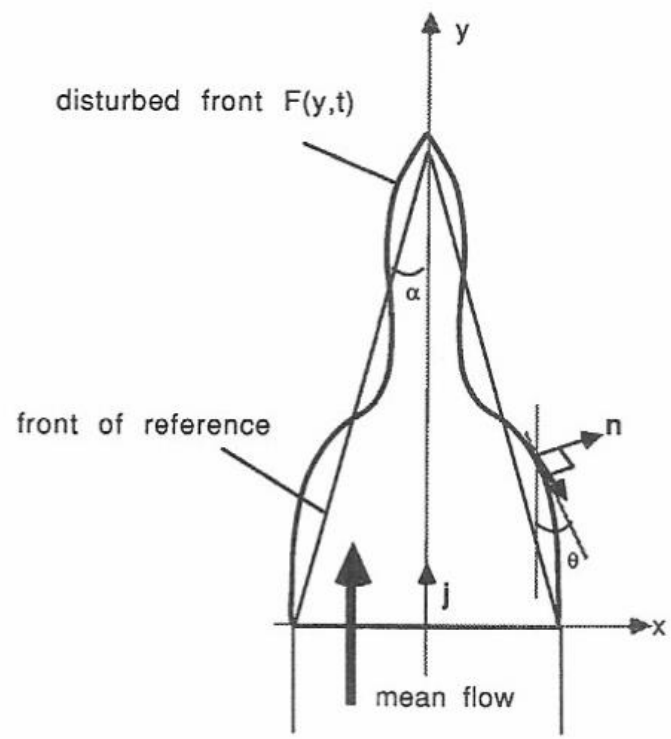

Fig. 13. Geometric description of the steady and unsteady theoretical fronts. 
The theoretical kinematic approach to the front deformation, is based on the following principal assumptions:

1. The velocity field does not appear to be a function of the front position.

2. The relative normal burning speed $v_{L}$ is uniform. Thus the front (disturbed or not) is characterized by the same relative normal burning speed - locally plane and adiabatic - which remains uniform if the unburned gases are at a fixed temperature, pressure, and concentration. The effects of the curvature and the stretching on $v_{L}$ are assumed to be negligible. This implies in particular that the velocity field, within the flame thickness, is not subjected to any local change, due to the presence of curvatures.

3. The flame is considered as an interface.

The flame is assumed to be a surface constantly attached to the edge of the burner, with the equation

$$
x=F(y, t)
$$

where $x$ represents the vector radius with respect to axisymmetrical flows. In the reference state, this evolution equation is written

$$
x=R-y \tan \alpha
$$

where $R$ is the radius of the burner and $\alpha$ is the half-angle at the apex of the conical front, the

sine of which is equal to the ratio $v_{L} / V_{0}$.

The unit normal to the instantaneous point of the flame, directed towards the burned gases, is at an angle $\theta$ to the horizontal (Fig. 13). We have

$$
\partial F / \partial y=-\tan \theta
$$

$$
v_{\perp}=\mathbf{v} \cdot \mathbf{n}=U \cos \theta+V \sin \theta
$$

and, if $w$ is the normal flame speed,

$$
w=\cos \theta \partial F / \partial t
$$

The parameters $U$ and $V$ represent the coordinates of the unburned gases velocity $\mathbf{v}$ projected in the polar coordinate system.

The dissipative phenomena in the gases are negligible upstream and downstream of the surface. Thus the only balance equation to be considered at the interface crossing is that of the mass conservation under the uniform burning velocity assumption:

$$
\left[\rho\left(v_{\perp}-w\right)\right]_{\mathrm{upstream}}=\left[\rho\left(v_{\perp}-w\right)\right]_{\mathrm{downstream}}=\mathrm{const}
$$

Or

$$
v_{\perp}-w=v_{L}
$$

With the notations introduced above, this leads to the new following expression: 


$$
\partial F / \partial t=U+V \tan \theta-v_{L} / \cos \theta
$$

From Eqs. 3 and 7 the fundamental equation is deduced:

$$
\partial \theta / \partial t+\left(V-v_{L} \sin \theta\right) \partial \theta / \partial y+U^{\prime} \cos ^{2} \theta+V^{\prime} \sin \theta \cos \theta=0
$$

$U^{\prime}$ and $V^{\prime}$ are the derivatives of the components $U$ and $V$ with respect to $y$ at constant $t$ and taking Eq. 2 into account. Provided with the boundary conditions indicating that $x=R$ for $y=0$ whatever the $t$ value, the functions $\theta(y, t)$ then $F(y, t)$ can be determined.

We introduce the nondimensional kinematic disturbance as a variation $\psi$ from the reference steady uniform flow $V=(1+\psi) V_{0}$, with $\psi=a \sin [2 \pi f(t-y / c)]$.

$\psi$ is a progressive wave of kinematic disturbance, propagating at the speed $c$, with a frequency $f$ such that the wavenumber $k$ is equal to the ratio $2 \pi f / c$. [ The case $\psi=\psi(t)$, which is a velocity disturbance propagating at quasi-sonic speed, has been also studied in Refs 8 and 10.]

The computation is made with nondimensional variables, relating the length to $R$, the velocity to $V_{0}$, and the time to $R / V_{0}$. The phase velocity $\omega$ is equal to $2 \pi f$.

The horizontal velocity component $U$ under the assumptions of incompressibility and axisymmetry is

$$
U=a k x \cos (w t-k y) / 2 \text {. }
$$

The complete analytical solution is that of the small disturbance case, that is, $a<<1$; hence the input of linearized variables:

$$
\begin{gathered}
\theta=\alpha+a \theta_{1}+O\left(a^{2}\right), \\
x=x_{0}+a x_{1}+O\left(a^{2}\right) .
\end{gathered}
$$

This velocity field does, in fact, verify the Euler equation of the order $O\left(a^{2}\right)$.

From Eq. 7 and the boundary conditions, the nondimensional expression $x$ of the front deformation is extracted (calculations are made in de tails in Ref. 8):

$$
\begin{aligned}
& x=1+y \tan \alpha+a x_{1}+O\left(a^{2}\right), \\
& x_{1}=-2\left\{A \cos \left(\omega \mathrm{t}-\omega Y / 2\left(1 / \mathrm{c}_{1}+1 / \mathrm{c}_{2}\right)\right),+\mathrm{B} \sin \left(\omega t-\omega Y / 2\left(1 / \mathrm{c}_{1}+1 / \mathrm{c}_{2}\right)\right\}\right. \\
& \sin \left(\omega Y / 2\left(1 / \mathrm{c}_{1}-1 / \mathrm{c}_{2}\right)\right)-Y C \sin \left(\omega t-w Y / c_{1}\right)
\end{aligned}
$$

with $A=k / 2(\omega-k \cos \alpha), \mathrm{B}=\tan \alpha\left(\omega-k \cos ^{2} \alpha /(\omega k \cos \alpha)^{2}=2 A h(\alpha) \tan \alpha / k\right.$, $C=A \tan \alpha, Y=y / \cos \alpha$, the ordinate of point of the front in an inclined system attached to the reference front, $c_{1}=\omega / k \cos \alpha$, and $c_{2}=\omega \cos \alpha / \mathrm{k}$.

A schematic representation of the speeds $c_{1}$ and $c_{2}$ is shown in Fig. 14. $c_{1}$ is the propagation velocity necessary for a flow disturbance covering the vertical distance $y$ at the velocity $\omega / k$, to cover the distance $Y$ along the front; $c_{2}$, is the driving velocity of the disturbance, tangentially to the reference flame front. 


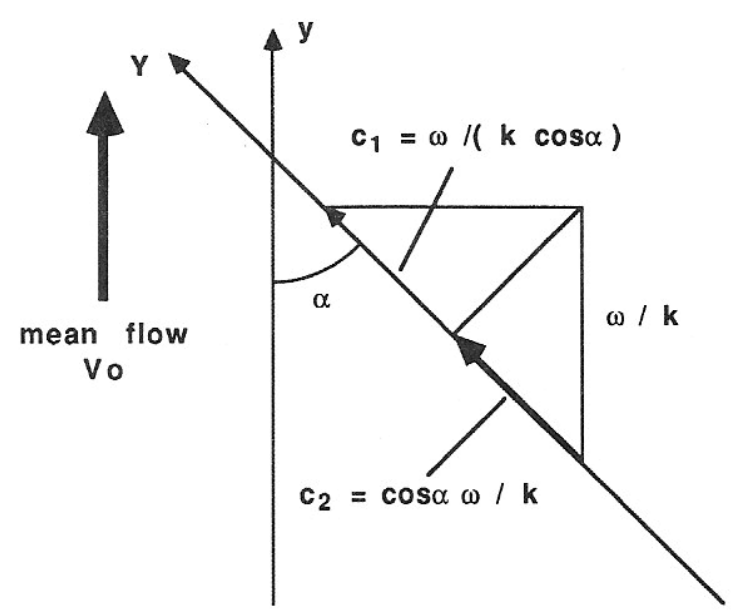

Fig. 14. Schematic representation of the wave speeds $c_{1}$ and $c_{2}$.

The front is thus deformed under the action of a transverse disturbance, a superposition of three single progressive waves expressed by $\mathrm{x}=\sin 2 \pi f(t-Y / e)$, each modulated by a fixed envelope. The space-time deformation of the front results from both local instantaneous disturbances located near the point of the front and disturbances that, starting at the base of the front, are convected along the front.

If we assume $\omega$ and $k(\omega)$ known a priori, the function $h(\alpha)$ remains bounded except for the two points $\alpha=0$ and $\alpha=\operatorname{arcos}(\omega / k)$ which are singular, respectively, in the two cases $k \rightarrow \omega$, and $k>\omega$. Thus for a flame high enough ( $Y$ large) or sufficiently wrinkled ( $k$ large), the function $Y C \sin \left(\omega t-\omega Y / c_{1}\right)$ becomes predominant and the propagation speed $c_{1}$ predominates. For long amplitudes of undulations ( $k$ small) and small flames, the term

$2 B \sin \left(\omega t-\omega Y / 2\left(1 / c_{1}+1 / c_{2}\right)\right.$ is the most important. The predominant propagation speed is $2 c_{1} c_{2} /\left(c_{1}+c_{2}\right)$. In the other cases, the three functions have a similar influence.

The computation of the absolute flame speed $\omega$, equal to $a \cos \alpha \partial x_{1} / \partial t+O\left(a^{2}\right)$, indicates the absence of steady nodes, characterized by a speed $w$ equal to zero whatever the $t$ value, except the anchorage point $(x=1, y=0)$, contrary to the case $\Psi=\Psi(t)[8,10]$.

There are two distinct propagation speeds, $c_{1}$ and $2 c_{1} c_{2} /\left(c_{1}+c_{2}\right)$ each of which defines a characteristic wavelength $\lambda_{1}$ and $\lambda_{2}$, equal to $c_{1} / f$ and $2 c_{1} c_{2} / f\left(c_{1}+c_{2}\right)$ Thus from a direct measurement of the wrinkling wavelength on the flame front, it is not possible to get back to $\lambda_{1}$ and $\lambda_{2}$. This calculation makes it possible to analyze the behavior of the front regardless of dispersion law $k=k(\omega)$. In particular an analysis of the case $k=\omega$, corresponding to a propagation at the mean flow velocity, is developed in Ref. 8.

\section{COMPARISON BETWEEN THE THEORETICAL AND EXPERIMENTAL RESULTS: CONCLUSION}

The comparison of experiment with theory is possible since the experimental conditions are included in the restricted field (frequency, intensity) of the disturbance, determined under the hypothesis of a propagation at the mean flow velocity, limiting the validity of the linearized model [8].

The theoretical reference configuration has been chosen by taking the ideal cone tangent to the actual flame front. Thus we have introduced in the computation of the front the numerical conditions based on the experiment and listed in Table 4.

Although we have not explicitly taken into account the influence of the flame upon the flow, and in particular the gas expansion [33], the characteristic values of the disturbed 
velocity field (frequency $f$, amplitude $a$, wavenumber $k$, speed $\langle\bar{V}\rangle$ ) introduced in the theoretical computation, correspond to those measured in the presence of the flame, and are therefore a priori influenced by the retroactive hydrodynamic effects of the flame on the flow of the unburned gases. For instance, compare the values of $k$ and $\langle\bar{V}\rangle$ obtained with and without flame (Figs. 4 and 6).

\section{TABLE 4}

Numerical Conditions Based on the Experiment (Test 2)

\begin{tabular}{lcc}
\hline$R(\mathrm{~mm})$ & 0.265 & 11 \\
$\alpha(\mathrm{rd})$ & 35.2 & 0.26 \\
$f(\mathrm{~Hz})$ & 2.27 & 2.29 \\
$V_{0}=\langle\bar{V}\rangle(\mathrm{m} / \mathrm{s})$ & 8.5 & 7.6 \\
$a=\sqrt{2}\left(v^{\prime} / \bar{V}\right)_{y=5 \mathrm{~mm}}(\%)$ & 0.855 & 1.91 \\
$k\left(\mathrm{~cm}^{-1}\right)^{a}$ & $\begin{array}{l}{ }^{a} k \text { is the slope of the curve of the interpolation at a given } \\
{ }^{a} k \text { is the points of the Fig. 4 with flame. }\end{array}$
\end{tabular}

An example of the theoretical and experimental front profiles, obtained by processing images, is plotted in Figs. 15 and 16. The comparison of the results at $35.2 \mathrm{~Hz}$, just as at 70.4 $\mathrm{Hz}$, indicates a very good agreement between experiment and theory, since the overall shape of the front is maintained. As might be expected, the most important differences appear at the base and the top of the front. In fact here, the curvature and stretching effects are more important and modify the value of the normal burning speed, whose variation is not considered in the calculations.
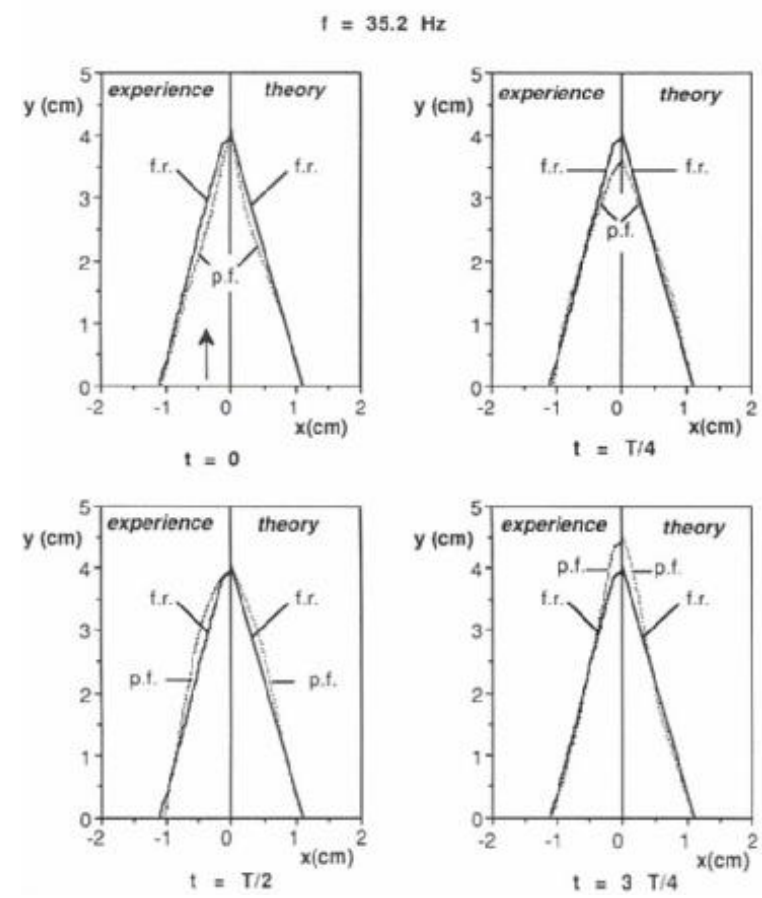

Fig. 15. Comparison of the theoretical fronts with the experimental fronts over a cycle at $35.2 \mathrm{~Hz}$. p.f., perturbed front; f.r., reference front. 

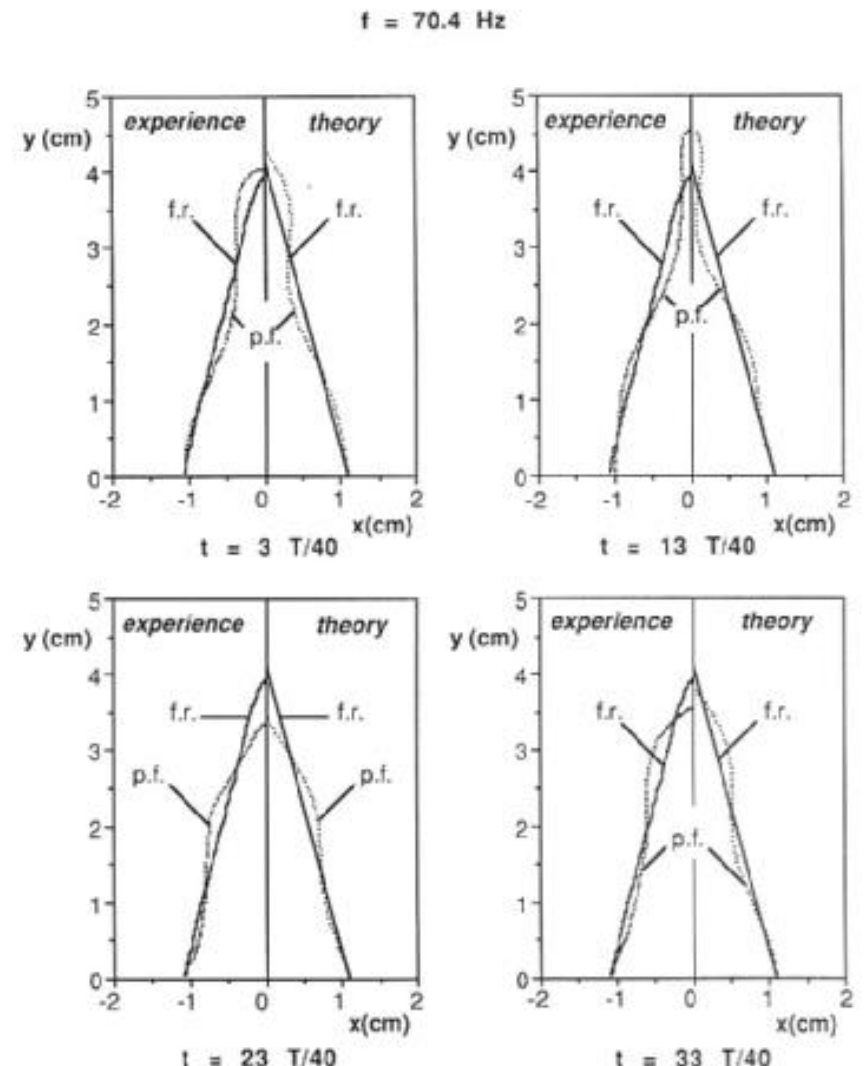

Fig. 16. Comparison of the theoretical fronts with the experimental fronts over a cycle at 70.4 Hz. p.f., perturbed front; f.r. reference front.

It can be considered that the experimental propagation of the deformation can be well represented by the theoretical model, and in particular by the simultaneous existence of the two velocities $\mathrm{c}_{1}$ and $\mathrm{c}_{2}$, practically equal to $(\bar{V}) / \cos \alpha$ and $(\bar{V}) \cos \alpha$ according to the experimental results of the section on Unburned Gases relative to the propagation speed at the mean flow velocity. In the case of high flames, the model shows that the apparent predominant velocity is $V_{0} / \cos \alpha$. The propagation speed is therefore proportional to the mean velocity $V_{0}$ of the flow. This phenomenon is mentioned in other theoretical and experimental works [1-6].

The comparison of the experimental and theoretical temporal curves of the front surface area, while confirming this agreement, shows some differences (Fig. 17). It would seem that the maxima have been undervalued. Indeed it has been demonstrated experimentally that the maximum areas are related to a maximum elongation of the flame at the top, and the assumption of a uniform normal burning velocity $v_{L}$ introduced in the computation quite obviously prevents the existence of such phenomena. Moreover, Fig. 17 shows an overvaluation of the theoretical minimum area $S_{\min } / S_{0}$ due to the truncation limited to the order $O\left(a^{2}\right)$ beyond the $v_{L}$ invariability assumption; the discontinuity of the flame curve appears as soon as the phenomenon of "pinching" occurs. The mean area $S_{T}$ calculated over a cycle is equal to the steady area $S_{0}$ at $35.2 \mathrm{~Hz}$, but at $70.4 \mathrm{~Hz} S_{T}$ exhibits a slight difference from $S_{0}$. This difference observed at $70.4 \mathrm{~Hz}$ can certainly be explained by the linearized method which is near its limit of validity as suggested in Ref. 8. On the other hand the equality between the two areas $S_{T}$ and $S_{0}$ has been confirmed experimentally at $35.5 \mathrm{~Hz}$ as well as at $70.4 \mathrm{~Hz}$. 

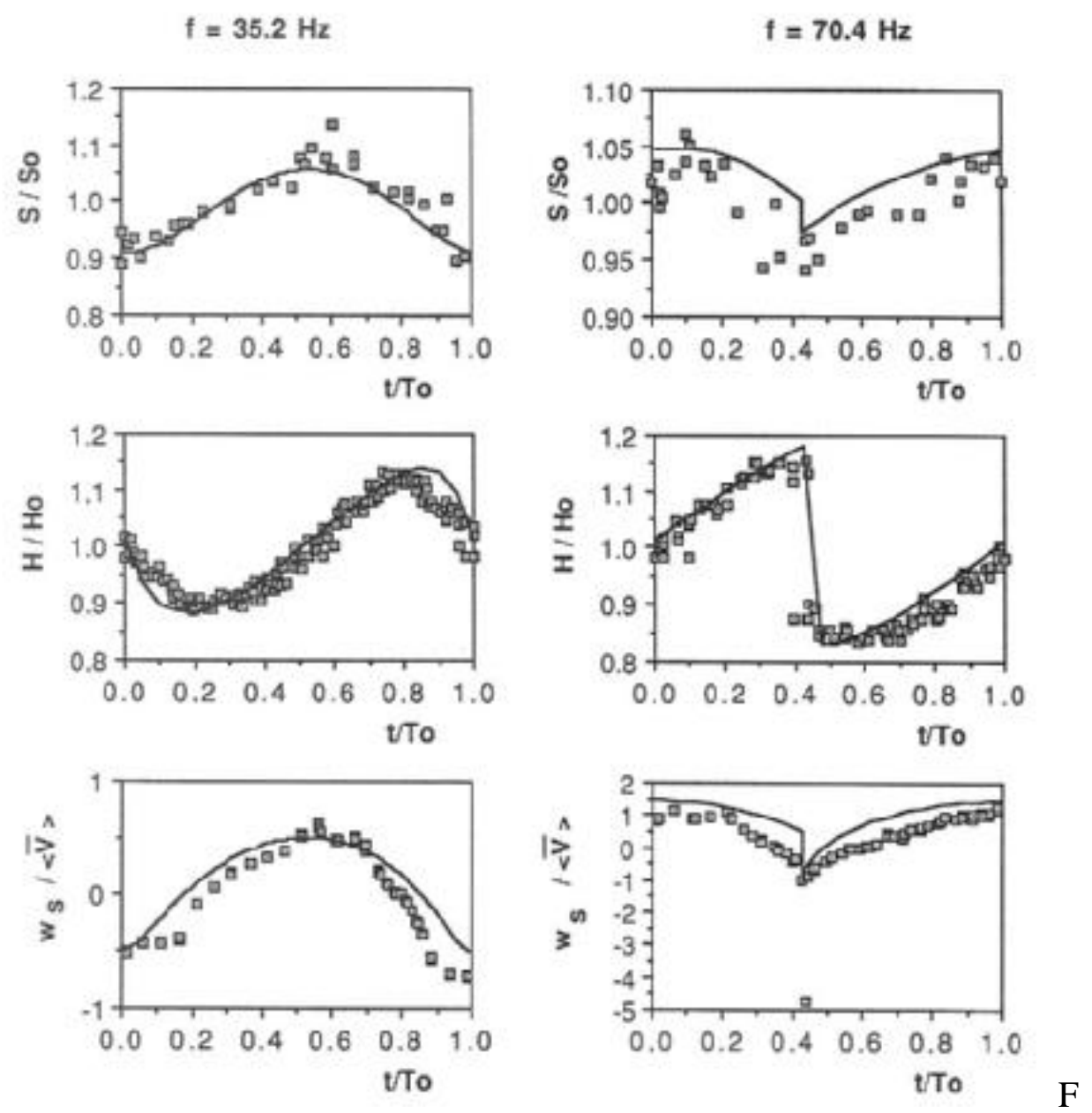

Fig. 17. Comparison of die theoretical area, height, anti tip velocity of the front (-) with the experimental values (.) over a cycle.

In Fig. 17, we have also plotted the nondimensional curves of the height $H(t)$ and the speed at the top $w_{\mathrm{s}}(t)$ The theoretical expression of the speed $w s$ is equal to $w\left(y_{\mathrm{s}}, t\right) / \sin \alpha$ at the first order of the asymptotic development as shown in Ref. 8. The reference height (experimental and theoretical) and the flow speed $\left(\langle V\rangle=V_{0}\right)$ are used to obtain nondimensional variables.

The curves of height evolution show a good agreement between experiment and theory The elongation and collapse phenomena are correctly represented by the model. A more accurate study shows some differences: at $35.2 \mathrm{~Hz}$, the theoretical front elongation duration is longer than that which is obtained experimentally. This causes an offset of the extrema $H_{\max }$ and $H_{\min }$ and a more rapid collapse of the front. At $70.4 \mathrm{~Hz}$, this difference is strongly reduced by the abrupt nature of the collapse. In both cases, the minimum height zone seems more spread out on the experimental curve than on the theoretical curve. The actual top of the flame has therefore a less rapid response to the collapse phenomenon than in the model. On the other hand, the amplitude of the flapping $\left(H_{\max }-H_{\min }\right) / H_{0}$ has been studied theoretically. The theoretical curve $\left(H_{\max }-H_{\min }\right) / H_{0}$ versus $f$, for a given value $a$, presents a curve with a maximum [8]. This represents a filter phenomenon for the highest frequency values. This behavior has been observed experimentally, and in particular the maximum of the flapping for the test 2 conditions is obtained in the vicinity of $70.4 \mathrm{~Hz}$.

The theoretical curves of the top speed over a cycle are in good agreement with the experimental velocity evolution. Overall, a quasi-systematic increase of the theoretical values over the experimental ones is observed. This difference cannot be only explained by the 
velocity measurement process. The assumption of a uniform vertical mean field should contribute to this translation. At $70.4 \mathrm{~Hz}$, the negative velocity peak is very much undervalued. The assumptions of invariance of the mean velocity field and of invariability of the normal burning speed probably hide the occurrence of these high values of $w_{\mathrm{s}}$.

This purely kinematic model of small disturbances has indeed demonstrated the predominant characteristics of the front deformation as much on a global level (form of the profiles of the front, surface variation) as locally at the top. The agreement of the experimental and theoretical results confirms the important role played by the kinematics of the unburned gas flow on the behavior of the front.

In the future it is necessary to extend the calculations in order to consider the variations of the burning speed with curvature and stretching. If this approach based on a method of small perturbations gives some information about the deformation process of the front, it would be useful then to develop a numerical scheme to complete the analysis in the presence of high perturbation amplitudes.

This study has provided the following information about the response of a premixed flame to an external sinusoidal low-frequency perturbation:

1. The front oscillates at the same frequency as the perturbation frequency.

2. The visualized thermodiffusive thickness at the top is unchanged in comparison to the laminar case: with only first-order consideration the perturbation does not seen to modify the inner structure of the flame.

3. The deformation of the front is due to progressive waves originated at its base and then convected along it proportionally to the flow velocity $\langle\bar{V}\rangle$. Two speeds of propagation appear, $\langle\bar{V}\rangle \cos \alpha$ and $\langle\bar{V}\rangle / \cos \alpha$, the latter of which predominates for sufficiently long fronts.

4. The flame is subjected during a cycle to large elongations, leading to important instantaneous variations of the front area $(25 \%$ at $35.2 \mathrm{~Hz}, 11 \%$ at $70.4 \mathrm{~Hz})$ and of the normal burning speed at the top (from 0.5 to 2 times the laminar value of the normal burning speed at the top). But on average the area is equal to the area of the reference laminar flame. This suggests that the stretching and curvature effects are negligible on average, even if locally they may be particularly important like at the top.

\section{REEERENCES}

1. Escudié, D., Lecture Notes Eng. Springer-Verlag, 40:323 (1989).

2. Herzberg, J. R., et al., Combust. Sci. Technol., 38:205-216 (1984).

3. Boyer. L., and Quinard, J., Combust. Flame 82:51-65 (1990).

4. Peterson, R E., and Emmons, H. W., Phys. Fluids 4:456-464 (1961).

5. Markstein, G. H., Combustion, Flame and Explosion Phenomena, 3rd Symposium, 1952, pp. 162-167.

6. De Soete, G., Rev. l'I.F.P. Anna. Combust. Liq., XIX:766 (1964).

7. Valk, M., Combust. Flame 41:251-260 (1981).

8. Baillot, F., Ph.D. thesis, University P. et M. Curie, Paris VI, March 1989.

9. Hoffman-Berling, E. et al., Nineteenth Symposium (International) on Combustion, The combustion Institute, Pittsburgh, 1982, pp. 433-439.

10. Baillot, F., C.R.A.Sc. 309:1649-1652, série II (1989).

11. Roginski, O. G., Sov. Phys. Acoust. 7:107-122 (1961). 
12. Blackshear, P. L., Combustion, Flame, and Explosion Phenomena, 4th Symposium, 1953, pp. 553-566.

13. Valk, M., Combust. Flame 41:251-260 (1981).

14. Poinsot, T. and Candel, S., Mathematical Modeling in Combustion and Related Topics, NATO ASI Series, E140, 1988, pp. 185-215.

15. McIntosh, A. C., Combust. Sci. Technol., 49: 143 (1986).

16. Hedge, U. G., et al., Combust. Sci. Technol., 55:125-138 (1987).

17. Hinze, J. O., Turbulence, McGraw-Hill, New York, 1959, p. 40.

18. Bradshaw, P., Turbulence, 2nd ed., Springer-Verlag, 1978, vol. 12, p. 21.

19. Van Tiggelen, A., et al., Oxydations et Combustion, Collection Science et Technique du Pétrole, 2. Publication IFP. 1968.

20. Williams, F. A., Combustion Theory, 2nd ed., Benjamin/Cummings, 1985.

21. Lewis. B., and Von Elbe, G., Combustion, Flames and Explosions of Gases. Academic, New York, 1951, pp.272-273.

22. Hirschfelder, J. O., Curtis, C. F., and Byron, R., Molecular Theory of Gases and Liquids, Wiley, New York, 1954.

23. Durox, D., Prud'homme, R., Baillot, F., and Bencheikh El Hocine, G., Joint Meeting of the British and French Sections of the Combustion Institute, 18-21 April 1989, Rouen, p. 183. 24. Durox, D., and Prud'homme, R., Seventh European Symposium on Materials and Fluids Sciences in Microgravity, 10-15 Sept. 1989, Oxford, p. 461.

25. Durox. D., Baillot. F., Scoufflaire, P., and Prud'homme, R., Combustion and Flame 82:66-74 (1990).

26. Dordain, J. J., and Lockwood, F. C., Fluid Sciences and Materials Science in Space (H. U. Walter, Ed.), Springer-Verlag. 1987, p. 306.

27. Boyer. L., Combust. Flame 39:321-323 (1980).

28. Boyer, L., and Searby, S., J. Appl. Phys. 6:2699-2707 (1986).

29. Baillot, F., Durox, D., and Prud'homme, R., International Specialities Meetings on the

Use of Computers in Laser Velocimetry, 18-20 May 1987, ISL, St. Louis, pp. 13/1-13/8.

30. Wagner, T., and Ferguson, C., Combust. Flame 59:267-272 (1985).

31. Baritaud, T., Ph.D. thesis, University P. et M. Curie, Paris VI, December 1983.

32. Dumont, J-P., Ph.D. thesis, University of Rouen, July 1987.

33. Landau, L., Acta Physicochim. URSS XIX:77-85 (1944). 